CASE REPORT

\title{
Case Report: An Unexpected Outcome of In Situ Fusion of the Calcaneo-Cuboid Joint
}

\section{Van Kerckhoven $J^{*}$, Van Beek $\mathbf{N}$ and Verfaillie $S$}

Department of Orthopedic Surgery, AZ Herentals, Belgium

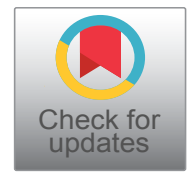

*Corresponding author: Jan Van Kerckhoven, Department of Orthopedic Surgery, AZ Herentals, Nederrij 133, 2200 Herentals, Belgium

\section{Introduction}

We present a case report where a necessary lengthening of the lateral column was performed after an in-situ fusion of the calcaneo-cuboid joint following an inversion injury to the ankle.

A low energy inversion injury of the ankle (ankle sprain) is one of the most common injuries presented at the emergency department and often results in ligamental injury of the anterior talo-fibular and calcaneo-fibular ligament. However, dislocating forces can proceed even more distally and also affect the foot at the calcaneo-cuboid level $[1,2]$. In some of these cases, such injuries can result in posttraumatic arthritis in the chopart-joint $[3,4]$.

The exact diagnosis of midfoot injuries is difficult to make based solely on conventional radiographs and thus many of these injuries are missed [5]. A lateral distraction injury to the calcaneo-cuboid joint (CC-joint) can cause a tear or avulsion of the bifurcate ligament and is known for causing long lasting pain symptoms. Some studies claim that up to $5 \%$ of all supination traumas of the ankle result in an avulsion of the anterior processes of the calcaneus (APC) [6]. In these cases, CT or MRI-imaging is required to make a correct diagnosis $[5,6]$.

Just as challenging as the diagnosis, is the treatment of these injuries. Good to excellent clinical outcome scores have been reported after functional treatment with weight bearing as tolerated for acute APC avulsion-fractures, but a prolonged time to return to sports was noted $[6,7]$. Degan, et al. and Lui, et al. reported successful treatment with lower leg cast immobilisation during 4-6 weeks with partial weight bearing and even recommend surgical fixation of bigger fragments $[8,9]$. In patients with persisting pain after an APC-avulsion, corticosteroid infiltration or excision of the fragment can be performed to relieve pain, although varying end results from a complete recovery to residual pain and swelling have been reported $[4,8,10,11]$.

Only a few treatment options remain if the injury has led to painful posttraumatic osteoarthritis of the midfoot. In general, arthritis of the midfoot joints is treated with fusion of these joints in order to eliminate motion and thereby pain [12]. However, this procedure is only performed when the complaints outweigh the loss of function. Within the foot, there is little space for joint preserving surgery or arthroplasty in end-stage arthritis. Arthrodesis is often well tolerated in the older population, who intrinsically have a stiffer joint, lower physical requirement and the fact that arthritic joints spontaneously tend to get stiffer with increasing age. Because of a higher intrinsic mobility in younger patients and especially women $[13,14]$, fusion of one joint might put adjacent joints under higher stress and may eventually put the fused joint under stress because of the loss of mobility.

\section{Case Description}

We present the case of a 21-year-old woman with no significant medical history. She was a non-smoker and worked in a supermarket. The patient suffered from an inversion trauma of her left ankle in December 2014. Her clinical symptoms where described as a painful, swollen lateral ankle. She did not tolerate weight

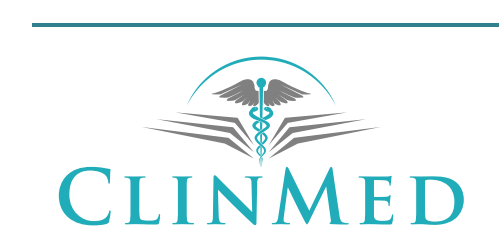

INTERNATIONAL LIBRARY

Citation: Van KJ, Van BN, Verfaillie S (2020) Case Report: An Unexpected Outcome of In Situ Fusion of the Calcaneo-Cuboid Joint. Int J Foot Ankle 4:046. doi.org/10.23937/2643-3885/1710046

Accepted: June 02, 2020: Published: June 04, 2020

Copyright: (C) 2020 Van KJ, et al. This is an open-access article distributed under the terms of the Creative Commons Attribution License, which permits unrestricted use, distribution, and reproduction in any medium, provided the original author and source are credited. 


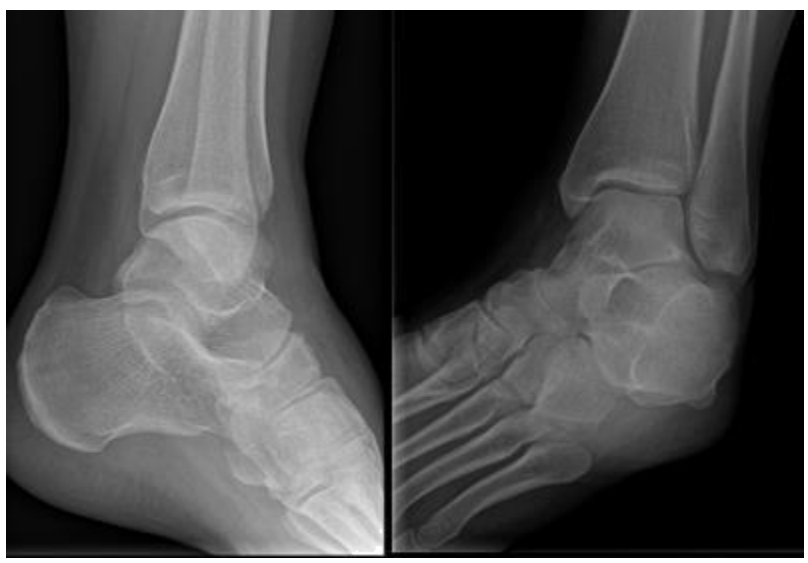

Figure 1: The initial radiographs from the left ankle taken at the emergency department in December 2014: To the left we see a medio-lateral projection and to the right we see the mortise-view into the talo-crural joint. These radiographs were protocoled as normal without posttraumatic injuries. A closer look however to the anterior process of the calcaneus shows a small avulsion. This is easily missed if not specifically looked for.

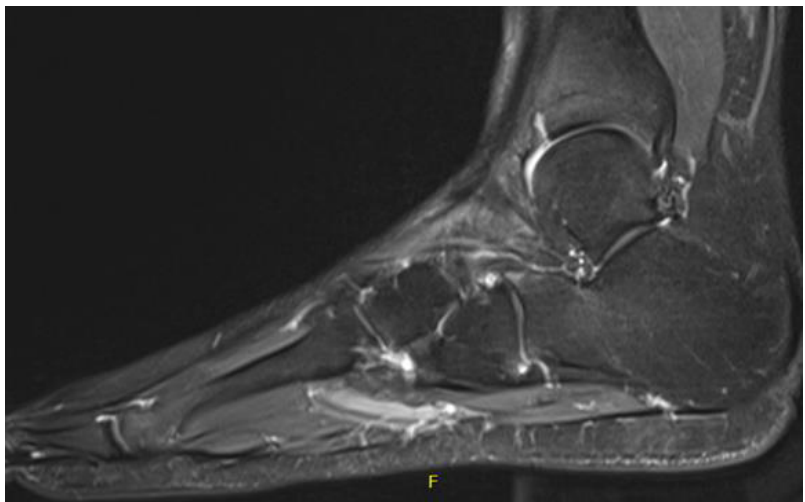

Figure 2: Sagittal view through the calcaneo-cuboid joint of the subsequent MRI taken in April 2015 shows the avulsion of the anterior process of the calcaneus.

bearing during first contact at the emergency department and plain radiographs taken at that time showed no fracture (Figure 1). The patient was diagnosed with a high-grade ankle sprain, the RICE principles where prescribed and the patient was sent home on crutches.

She was seen again 4 weeks later with persisting pain complaints, unable to fully bear weight and unable to work. Physiotherapy and brace immobilisation were prescribed for four months, but no improvement was seen. A subsequent MRI was taken, showing an avulsion of the dorsolateral process of the calcaneus (Figure 2). At 4 months after the initial trauma, a local infiltration of $40 \mathrm{mg}$ depot-medrol was given to alleviate symptoms, but no improvement was seen. A series of 3 more infiltrations with short intervals were tested 8 months later without result.

Two years after the initial trauma, early 2017, she presented at our clinic for a second opinion. She stopped working due to persisting ankle pain during weight-bearing. Clinical examination showed anterior-posterior in-

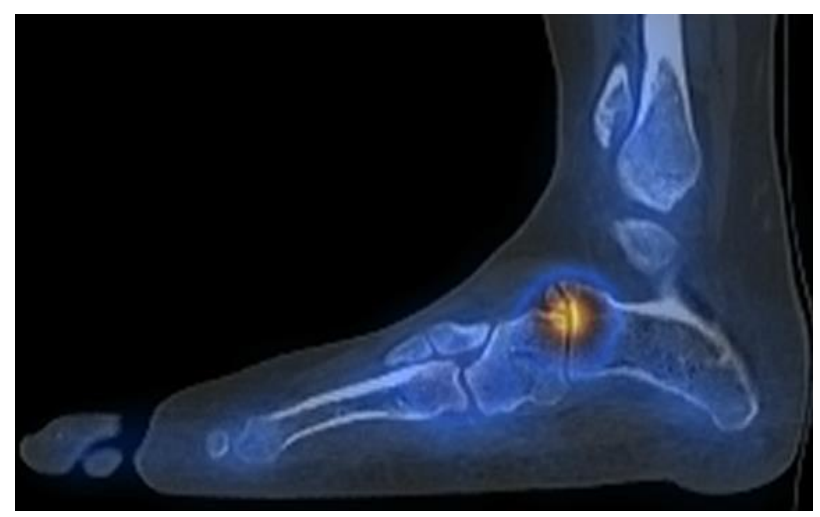

Figure 3: Sagittal view on SPECT-CT taken in January 2017, showing a non-union of the avulsion fracture and arthritis of the calcaneo-cuboid joint.

stability and pain located over the CC-joint. A SPECT-CT was taken, showing a hotspot over the CC-joint and a residual avulsion of the dorsolateral calcaneal fragment (Figure 3). The CC-joint also had a degenerative aspect.

We diagnosed post-traumatic arthritis of the CC-joint due to instability after avulsion of the bifurcate ligament between the calcaneus and the cuboid. Based on the clinical examination, SPECT-CT findings and longevity of the symptoms, an extirpation of the avulsed fragment was expected to fail. Therefore, the decision was made to perform a salvage procedure consisting of in-situ arthrodesis of the CC-joint with non-structural spongiosa graft taken from the proximal tibia.

The sinus-tarsi approach was used and arthritic destruction of the CC-joint in correlation with the SPECTCT was seen. The remaining cartilage and subchondral bone on both sides of the CC-joint was removed until bleeding spongiosa was present. The defect was filled with spongiosa taken from the proximal tibia and stabilised with a lateral bridging plate. The ankle was immobilised with an open cast for 10 days and a circular cast for 4 weeks with progressive weight bearing. Three months after surgery, the fusion showed a good position and progressive healing, although the patient still complained of weight-bearing pain.

Five months after surgery, her pain was identical to preoperatively and she could not stand for long periods of time. The wound had healed, and no signs of infection were visible. A CT-scan was taken, and progressive bone formation was present in the arthrodesis. Medially, a gap in the CC-joint was seen but no osteolysis around the screws was visible nor any failure of the hardware (Figure 4).

One year after surgery it was decided to remove the lateral bridging plate through the same incision. Peroperative, no signs of infection were seen, and the fusion was completely stable. After plate removal, the complaints remained unchanged. On SPECT-CT 14 and 22 months after arthrodesis (Figure 5), a persisting hotspot was seen. There were no signs of osteomyelitis, osteol- 


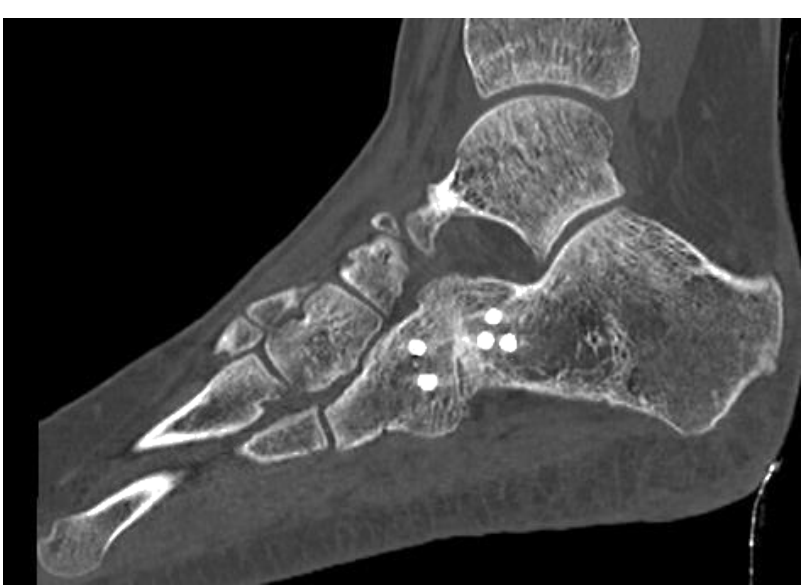

Figure 4: Sagittal view on the CT-scan taken 6 months after surgery, showing progressive consolidation of the calcaneo-cuboid joint and good position of the hardware.

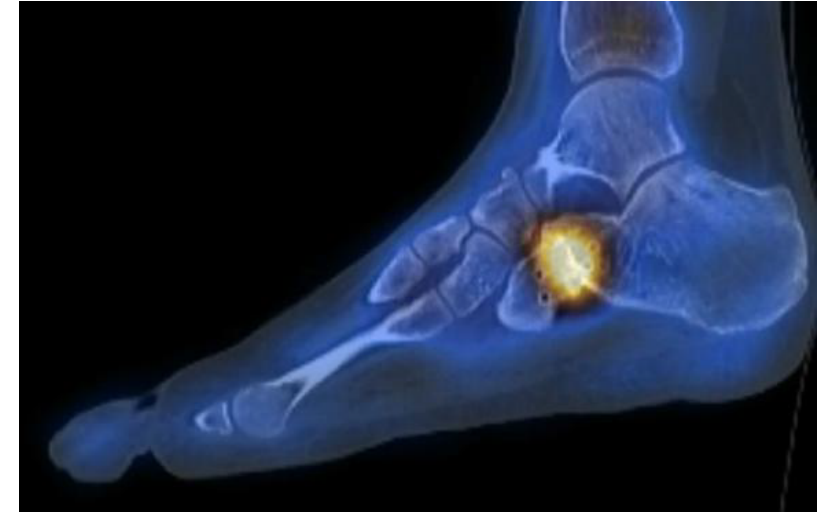

Figure 5: Sagittal view of the calcaneo-cuboid joint on SPECT-CT taken 22 months after arthrodesis and 10 months after hardware removal shows remaining activity within the arthrodesis.

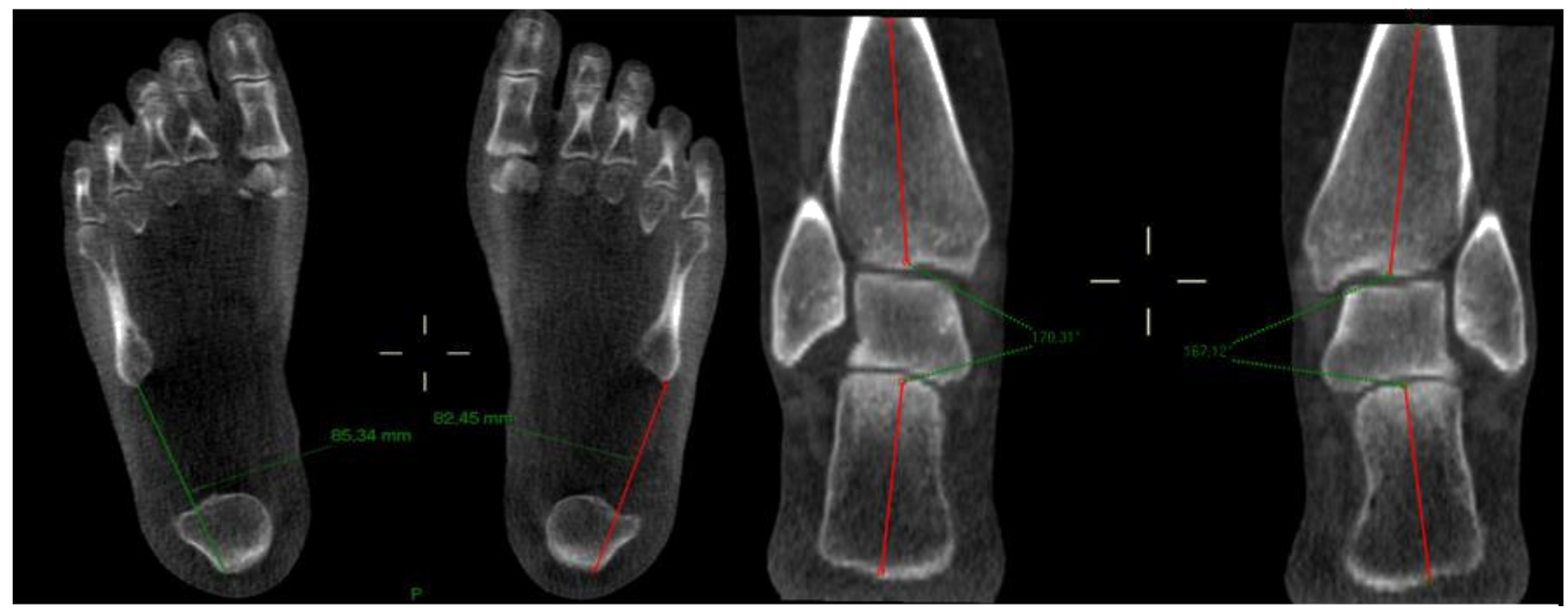

Figure 6: Weight-bearing CT with transverse view of both feet to the left and axial view at a $60^{\circ}$ angle of both heels to the right. Measuring the distance between the styloid process of the $5^{\text {th }}$ metatarsal and the most posterior part of the calcaneus reveals shortening between the lateral column in the left foot compared to the right foot. Comparing heel valgus left and right shows slightly increased valgus on the left side.

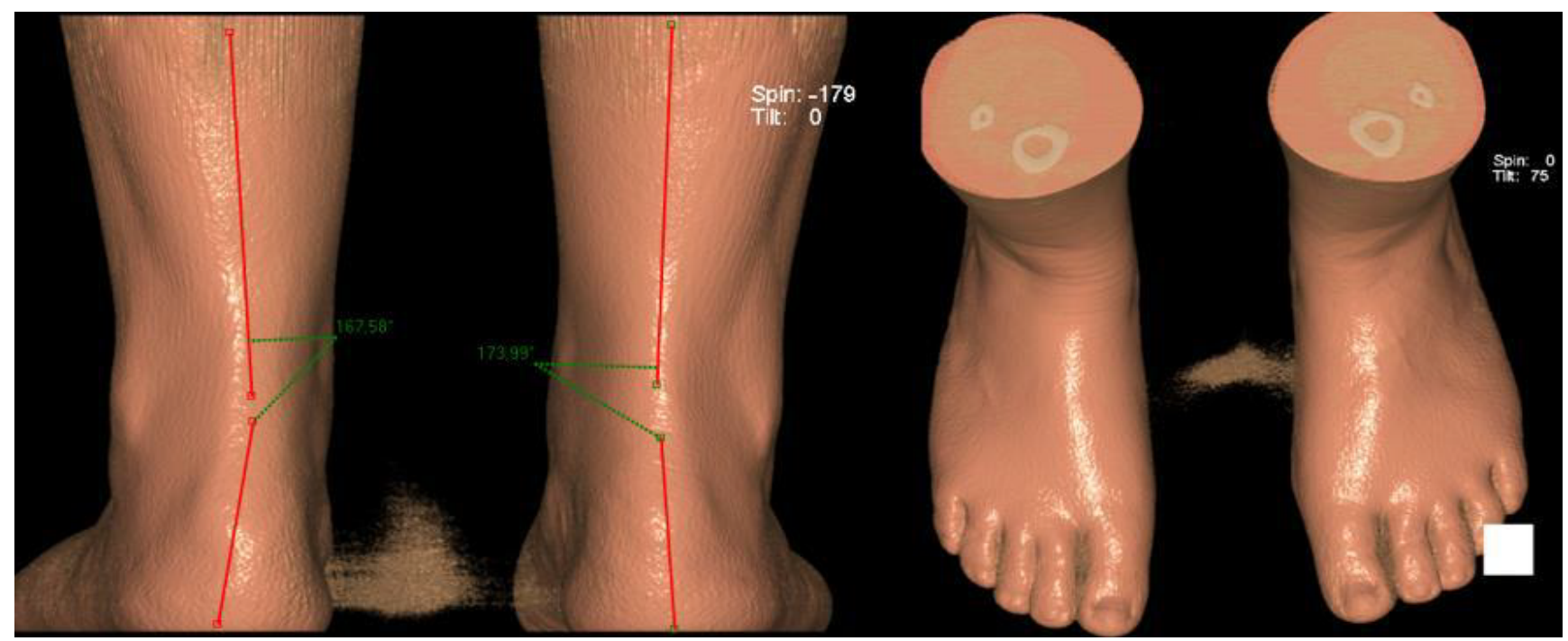

Figure 7: At the left: Posterior view on 3D-reconstruction of the feet shows increased heel valgus in the left heel $\left(12^{\circ}\right.$ left vs. $6^{\circ}$ right). At the right: View from above showing discrete increased forefoot abduction in the left foot. 
ysis or cortical discontinuation and the signal-intensity remained unchanged.

Clinical examination showed somewhat more heel valgus and forefoot abduction on the left side. In order to get a better understanding of the foot dynamics, a weight-bearing CT was taken and a shortening of the lateral column with $3 \mathrm{~mm}$ after fusion in-situ was diagnosed (Figure 6). The shortening of the lateral column had pushed the forefoot slightly in abduction and the calcaneus in valgus (Figure 7). The fusion of the CC-joint had shortened the lateral column and caused a subtle planovalgus deformity in the foot, thus increasing pressure through the lateral column.

A revision of the $\mathrm{CC}$-joint arthrodesis with a lengthening of the lateral column was proposed. A tricortical graft from the crista iliaca was used for the corrective lengthening osteotomy of the CC-joint. This procedure is comparable to a flexible flatfoot correction by lateral column lengthening (Figure 8). Five months after revision surgery, the patient finally started to improve clinically. She still experienced good and bad days, but swelling was getting better and less and less pain was

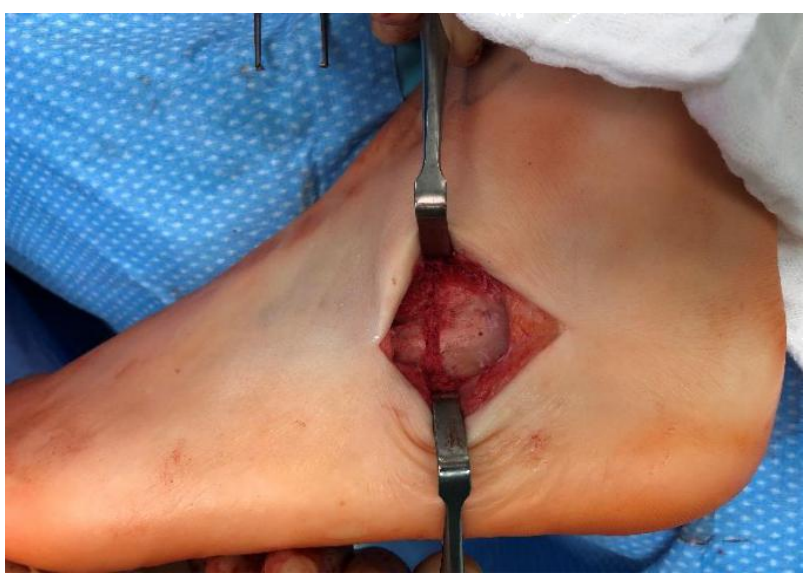

Figure 8: Peroperative photo of the lengthening arthrodesis of the calcaneo-cuboid joint through the sinus tarsi approach.

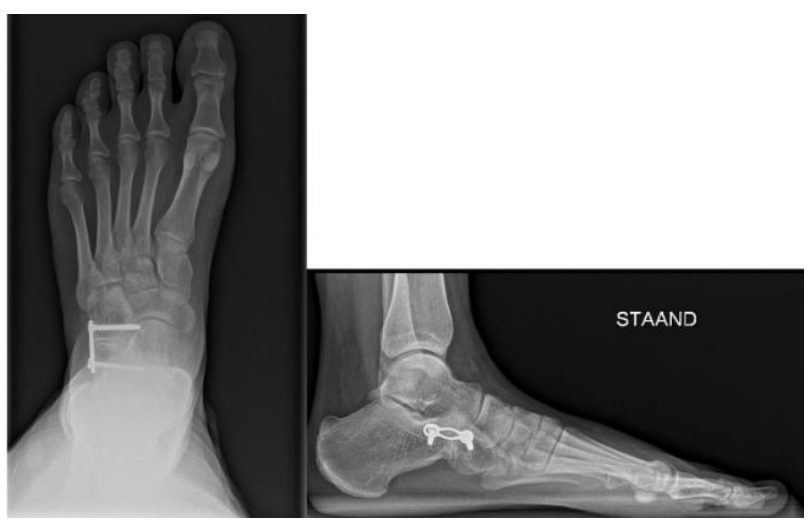

Figure 9: Weight bearing radiographs 5 months after revision arthrodesis with lengthening of the lateral column. Medio-lateral view at the right, cranio-caudal view at the left. felt during walking. She was infiltrated twice with depot-medrol in the sinus tarsi 5 and 7 months after surgery, with good effect. Weight bearing radiographs showed a good position of the arthrodesis (Figure 9). On clinical examination, her foot showed better statistics, with a symmetrical valgus of both heels and forefoot pronation. She could start working again part-time 5 months postoperative.

\section{Discussion}

The aim of this case report is to highlight the delicacy of foot statistics, especially in younger patients. The calcaneo-cuboid joint connects the hindfoot with the forefoot and is an essential structure during stance and gait. The midtarsal locking system and load transmission through the transverse midtarsal joints is quite complicated and has been described by several authors [15-17]. This, together with the fact that the foot's flexibility is gender and age dependent [13], supports our hypothesis that the fusion of one of the midtarsal joints has a greater impact on kinetics and kinematics of the foot in younger patients compared to older patients. In our case, we see that a shortening of the lateral column with only three millimetres after an in-situ fusion of the CC-joint, caused a subtle abduction and pronation of the forefoot and an increased valgus in the hindfoot. These changes, combined with the loss of elasticity through the CC-joint, increased the stress through the lateral column.

As we could only detect subtle changes on the weight bearing CT-scan, we think that the fused CC-joint didn't allow the foot to adopt a more pronounced planovalgus position as would be expected after shortening of the lateral column. Nonetheless, the force transmission through the lateral column was changed. The increased bone stress in the arthrodesis was visible with 2 SPECT-CT's, the first 14 months after arthrodesis and the second 22 months after arthrodesis. The intensity of the signal remained unchanged during this period. To the best of our knowledge, and after exclusion of other reasons for the remaining activity within the arthrodesis such as infection, hardware failure etc., the only explanation for the remaining symptoms was bone stress due to a slight increase in heel valgus. With a corrective lengthening osteotomy, a well-known treatment for flatfoot correction $[15,18]$, we aimed to restore the kinematics through the lateral column in order to decrease the bone stress.

\section{Conclusion}

The biomechanical model of the foot is complex and poorly understood. Surgical options in end-stage osteoarthritis are limited and often consist of arthrodesis of the painful joint. Unlike in older patients, we experience that arthrodesis of midtarsal joints is less tolerated in a younger patient population. Our hypothesis is that shock- and force transmission during stance and gait is 
a very delicate mechanism that depends on small but essential movements through the midfoot. Especially in younger patients, alterations within the foot kinetics and kinematics can significantly change the load distribution within the foot and may cause bone stress and pain. We therefore recommend to respect foot statistics to a maximum, especially within younger patients, and to reconstruct foot-physiognomy as good as possible.

\section{References}

1. Schepers T, van Schie-van der Weert EM, de Vries MR, van der Elst M (2011) Foot and ankle fractures at the supination line. Foot 21: 124-128.

2. Walter WR, Hirschmann A, Alaia EF, Garwood ER, Rosenberg ZS (2018) JOURNAL CLUB: MRI evaluation of midtarsal (Chopart) sprain in the setting of acute ankle injury. Am J Roentgenol 210: 386-395.

3. Van Dorp KB, De Vries MR, Van Der Elst M, Schepers T (2010) Chopart joint injury: A study of outcome and morbidity. J Foot Ankle Surg 49: 541-545.

4. Klaue K (2004) Chopart fractures. Injury 35: SB64-SB70.

5. Massen FK, Baumbach SF, Böcker W, Kammerlander C, Herterich V, et al. (2018) Fractures of the anterior process of the calcaneus-frequently overlooked injuries following ankle sprains. Unfallchirurg 121: 730-738.

6. Schepers T, Ginai AZ, Lieshout EMM, Patka P (2008) Demographics of extra-articular calcaneal fractures: Including a review of the literature on treatment and outcome. Arch Orthop Trauma Surg 128: 1099-1106.

7. Massen FK, Baumbach SF, Herterich V, Böcker W, Waizy $\mathrm{H}$, et al. (2019) Fractures to the anterior process of the calcaneus - Clinical results following functional treatment. Injury 50: 1781-1786.
8. Degan TJ, Morrey BF, Braun DP (1982) Surgical excision for anterior-process fractures of the calcaneus. J Bone Joint Surg Am 64: 519-524.

9. Hing Lui T (2011) Endoscopic excision of symptomatic nonunion of anterior calcaneal process. J Foot Ankle Surg 50: 476-479.

10. Halm JA, Schepers T (2017) Resection of small avulsion fractures of the anterior process of the calcaneus for refractory complaints. J Foot Ankle Surg 56: 135-141.

11. Dhinsa BS, Latif A, Walker R, Abbasian A, Back D, et al. (2018) Fractures of the anterior process of the calcaneum: A review and proposed treatment algorithm. Foot Ankle Surg 25: 258-263.

12. Kutaish H, Stern R, Drittenbass L, Assal M (2017) Injuries to the Chopart joint complex: A current review. Eur J Orthop Surg Traumatol 27: 425-431.

13. Nigg BM, Fisher V, Ronsky JL (1994) Gait characteristics as a function of age and gender. Gait \& Posture 2: 213-220.

14. By Sinclair J, Chockalingam N, Vincent H (2014) Gender differences in multi-segment foot kinematics and plantar fascia strain during running. The Foot and Ankle Online Journal 7: 2 .

15. Suckel A, Muller O, Langenstein $P$, Herberts $T$, Reize $P$, et al. (2007) Chopart's joint load during gait. In vitro study of 10 cadaver specimen in a dynamic model. Gait Posture 27: 216-222.

16. Brockett CL, Chapman GJ (2016) Biomechanics of the ankle. Orthop Trauma 30: 232-238.

17. Lundberg A, Goldie I, Kalin bo, Selvik G (1989) Kinematics of the ankle/foot complex: Plantarflexion and Dorsiflexion. Foot Ankle 9: 194-200.

18. Evans D (1975) Calcaneo-valgus deformity. J Bone Joint Surg $\operatorname{Br} 57:$ 270-278. 